\title{
Counting nondecreasing integer sequences that lie below a barrier
}

\author{
Robin Pemantle \\ University of Pennsylvania \\ Philadelphia, PA, 19104-6395 \\ pemantle@math.upenn.edu
}

\author{
Herbert S. Wilf \\ University of Pennsylvania \\ Philadelphia, PA 19104-6395 \\ wilf@math . upenn . edu
}

Submitted: Apr 16, 2009; Accepted: Apr 29, 2009; Published: May 11, 2009

Mathematics Subject Classification: 05A15, 60C05

\begin{abstract}
Given a barrier $0 \leq b_{0} \leq b_{1} \leq \cdots$, let $f(n)$ be the number of nondecreasing integer sequences $0 \leq a_{0} \leq a_{1} \leq \cdots \leq a_{n}$ for which $a_{j} \leq b_{j}$ for all $0 \leq j \leq n$. Known formulæ for $f(n)$ include an $n \times n$ determinant whose entries are binomial coefficients (Kreweras, 1965) and, in the special case of $b_{j}=r j+s$, a short explicit formula (Proctor, 1988, p.320). A relatively easy bivariate recursion, decomposing all sequences according to $n$ and $a_{n}$, leads to a bivariate generating function, then a univariate generating function, then a linear recursion for $\{f(n)\}$. Moreover, the coefficients of the bivariate generating function have a probabilistic interpretation, leading to an analytic inequality which is an identity for certain values of its argument.
\end{abstract}

\section{Introduction}

We are given a sequence $\mathbf{b}:=b_{0} \leq b_{1} \leq \cdots$ of nonnegative integers. For another sequence $\mathbf{a}:=a_{0} \leq a_{1} \leq \ldots$, we write $\mathbf{a} \preceq \mathbf{b}$ if $a_{i} \leq b_{i}$ for all $i$. Fix the upper sequence $\mathbf{b}$, which will be known as the barrier. Let $f(n)$ denote the number of finite sequences $0 \leq a_{0} \leq a_{1} \leq \cdots \leq a_{n}$ lying below the barrier.

The object of this paper is to find $f(n)$. We first find a generating function for $\{f(n)\}$, namely

$$
\sum_{n \geq 0} f(n) x^{n+1}(1-x)^{1+b_{n+1}}=1-(1-x)^{1+b_{0}} .
$$

The form of this function (mixed powers of $x$ and $1-x$ ) is somewhat unusual but also it suggests, particularly if we replace $x$ by $p$ and $1-x$ by $q$, that a probabilistic mechanism is operating as well as the combinatorial one. We then describe a family of random walks in the region below the barrier, the family having the property that the $n$th term in (1) 
above is the probability that a walk exits the region under the barrier at exactly the $n$th step. However, it is possible that with some positive probability a walk will never exit that region, in which case the left-hand side of (1) will be strictly less than the right-hand side. This apparent paradox is resolved by the observation that although (1) is always true in the ring of formal power series (therefore determining the numbers $f(n)$ uniquely by recursion), it is not necessarily true as an analytic equality; in fact it will be true analytically if and only if the probability is 1 that the walk eventually collides with the barrier.

\section{Summary of results}

When we wish to emphasize the role of $\mathbf{b}$, we will write $f(\mathbf{b} ; n)$ in place of $f(n)$. Further we let $\mathbf{a}_{\mid m}$ denote the truncation of $\mathbf{a}$ to $\left(a_{0}, \ldots, a_{m}\right)$, and extend the notation to write $\mathbf{a}_{\mid m} \preceq \mathbf{b}$ when $a_{i} \leq b_{i}$ for $0 \leq i \leq m$. Then formally, $f(\mathbf{b} ; n)$ is the number of sequences $\mathbf{a}_{\mid n}$ for which $\mathbf{a}_{\mid n} \preceq \mathbf{b}$. Equivalently, $f(\mathbf{b} ; n)$ is the number of integer partitions with $n$ parts whose Ferrers diagram is entirely contained in the diagram for $\mathbf{b}$, or the number of elements of Young's lattice that lie below $\mathbf{b}$.

An explicit formula for $f(\mathbf{b} ; n)$ is known. In fact, for the more general problem of counting sequences of length $n$ lying between two barriers, $\mathbf{a}$ and $\mathbf{b}$, Kreweras proved in 1965 [Kre65] that the number $f(\mathbf{a}, \mathbf{b} ; n)$ of nondecreasing integer sequences between barriers $\mathbf{a}$ and $\mathbf{b}$ is given by the following $n \times n$ determinant:

$$
f(n)=\operatorname{det}\left(\left(\begin{array}{c}
b_{i}-a_{j}+n \\
i-j+n
\end{array}\right)_{i, j=0, \ldots, n}\right) .
$$

Our first result is a recursion leading to a quadratic-time computation for $f(n)$ :

Theorem 1. The numbers $\{f(n)\}$ of nondecreasing integer sequences between zero and b satisfy the recurrence

$$
f(n)=(-1)^{n}\left(\begin{array}{c}
b_{0}+1 \\
n+1
\end{array}\right)+\sum_{0 \leq m<n}(-1)^{m+n+1}\left(\begin{array}{c}
1+b_{m+1} \\
n-m
\end{array}\right) f(m), \quad(n=0,1,2, \ldots) .
$$

This theorem follows from the following formal power series identity.

\section{Theorem 2.}

$$
1=(1-x)^{1+b_{0}}+\sum_{n \geq 0} f(n) x^{n+1}(1-x)^{1+b_{n+1}}
$$

in the ring $\mathbb{C}[x]$.

As a special case, we recover a formula in the case of a linear barrier due to $\mathrm{R}$. Proctor [Pro88] (see also [Sta99, (7.194) in exercise 7.101b]): if $b_{j}=r j+s$ then

$$
f(\mathbf{b} ; n)=\frac{s+1}{n+1}\left(\begin{array}{c}
s+(n+1)(r+1) \\
n
\end{array}\right) .
$$


For convergence of the sum (4) in the formal power series ring, it is necessary, as is indeed that case, that there be only finitely many summands for each monomal $x^{k}$. One may ask whether in fact (4) holds as an analytic identity. The probabilistic interpretation of the summands in (4), leading to the following result, is elaborated in the proof in Section 4.

Theorem 3. Fix a barrier $\mathbf{b}$ and a number $p \in(0,1)$. Let $q:=1-p$. For each $n$ and each sequence $\mathbf{a}:=\left(a_{0} \leq \cdots \leq a_{n}\right) \preceq \mathbf{b}$ with length $\ell(\mathbf{a})=n+1$, define the weight

$$
w(\mathbf{a}):=\frac{p^{n+1} q^{b_{n+1}+1}}{1-q^{1+b_{0}}} .
$$

Then

$$
\sum_{\mathbf{a}} w(\mathbf{a}) \leq 1
$$

where the sum is over all finite sequences, that is, over all $k>0$ and all $\mathbf{a}$ with $\ell(\mathbf{a})=k$. Furthermore, if we let $\theta:=\lim \inf b_{n} / n \in[0, \infty]$ then we can determine whether equality holds in (6) in nearly all cases, as follows.

(i) if $\theta<q / p$ then equality holds;

(ii) if $\theta=q / p$ then equality holds provided $\left|b_{n}-\theta n\right|=O(\sqrt{n})$;

(iii) if $\theta>q / p$ then equality fails.

\section{Combinatorial proofs}

First we will prove Theorem 2, after which Theorem 1 follows almost immediately.

Proof of TheOrem 2: We begin by identifying some elementary recursions. Fix the barrier sequence $\mathbf{b}$ and let

$$
c(m, j):=f\left(\mathbf{b}_{\mid m} ; j\right)
$$

count the class $\Gamma(m, j)$ of sequences $\mathbf{a} \preceq \mathbf{b}$ of length $m+1$ for which $a_{m}=j$. We define $c(m, j):=0$ if $m<0$ or if $j>b_{m}$. Partitioning $\Gamma(m, j)$ according to the value of $a_{m-1}$ gives a disjoint union $\bigcup_{i} \Gamma(m-1, i)$. The set $\Gamma(m-1, i)$ is empty when $i>j$, whence

$$
c(m, j)=\sum_{i=0}^{j} c(m-1, i) .
$$

In particular, setting $j=b_{m}$,

$$
f(m-1)=c\left(m, b_{m}\right) .
$$

Next, we partition $\Gamma(m, j)$ into $A \cup B$ where $A$ is the set of sequences a with $a_{m-1}=a_{m}=j$ and $B$ is the set of sequences with $a_{m-1}<a_{m}=j$. The set $B$ is in bijection with the set 
$\Gamma(m, j-1)$ via the map that changes $a_{m}$ from $j$ to $j-1$ and fixes $a_{i}$ for $i<m$. Clearly, $|A|=c(m-1, j)$. This implies the relation

$$
c(m, j)=c(m-1, j)+c(m, j-1)
$$

for every $m>0$ and $0<j \leq b_{m}$. Checking the boundary case $j=0$, we have $c(m, 0)=1$ for $m \geq 0$ and zero otherwise, so (9) holds for $j=0$ as long as $m \neq 0$. When $m=0$, we have $c(0, j)=1$ if $0 \leq j \leq b_{0}$ and zero otherwise, whence (9) holds for $m=0$ as long as $j \notin\left\{0, b_{0}+1\right\}$. When $j \geq b_{m}+2$, all the terms of (9) are zero and the relation holds vacuously. Finally, for any $m \geq 0$ and $j=b_{m}+1$ we have

$$
c(m, j)-c(m-1, j)-c(m, j-1)=-c\left(m, b_{m}\right)=-f(m-1)
$$

by (8). Define a bivariate generating function

$$
C(x, t):=\sum_{m, j \geq 0} c(m, j) x^{m} t^{j} .
$$

The relations (9) and exceptions (10) imply that

$$
(1-x-t) C(x, t)=1-t^{1+b_{0}}-\sum_{m>0} f(m-1) x^{m} t^{1+b_{m}} .
$$

The kernel method (see, e.g., [BMP00, FS08]) suggests the substitution $t=1-x$. On both sides of (11) the power of $t$ appearing in any monomial $x^{m} t^{j}$ is bounded by $b_{m}+1$, hence the substitution is valid in the ring of formal power series and yields

$$
0=1-(1-x)^{1+b_{0}}-\sum_{m>0} f(m-1) x^{m}(1-x)^{1+b_{m}} .
$$

With $m=n+1$, this is Theorem 2 .

Proof of TheOrem 1: For $k \geq 0$, the coefficient of $x^{k+1}$ on the right-hand side of (4) is known to vanish. But this coefficient is equal to

$$
(-1)^{k+1}\left(\begin{array}{c}
b_{0}+1 \\
k+1
\end{array}\right)+\sum_{m=0}^{k} f(m)(-1)^{k-m}\left(\begin{array}{c}
b_{m+1}+1 \\
k-m
\end{array}\right) .
$$

Solving for $f(k)$ gives

$$
f(k)=-\left[(-1)^{k+1}\left(\begin{array}{c}
b_{0}+1 \\
k+1
\end{array}\right)+\sum_{m=0}^{k-1} f(m)(-1)^{k-m}\left(\begin{array}{c}
b_{m+1}+1 \\
k-m
\end{array}\right)\right]
$$

which is Theorem 1 with the variable $k$ in place of $n$.

To prove (5), let $F(x):=\sum_{n>0} f(n) x^{n}$ be the generating function for $\{f(n)\}$. Again, substitute $t=1-x$ in (11); the left-hand side is again zero, while the choice of $b_{n}=r n+s$ makes the right-hand side into

$$
1-(1-x)^{s+1}-x(1-x)^{r+s+1} F\left(x(1-x)^{r}\right) .
$$


There is a unique formal power series $X(y)$ with no constant term such that $X(y)(1-$ $X(y))^{r}=y$. From (12) we get

$$
F\left(x(1-x)^{r}\right)=\frac{1-(1-x)^{s+1}}{x(1-x)^{r+s+1}} .
$$

Composing formally with $X$ we obtain

$$
F(y)=\frac{1-(1-X(y))^{s+1}}{y(1-X(y))^{s+1}}
$$

Thus,

$$
f(n)=\left[y^{n}\right] F(y)=\left[y^{n+1}\right](y F(y))=\left[y^{n+1}\right]\left(\frac{1}{(1-X(y))^{s+1}}-1\right)
$$

which we may now evaluate via Lagrange inversion. The following form of the Lagrange inversion formula may be found in [Wil94].

Lemma 4. Let $\phi$ be formal power series in $x$ with $\phi(0)=1$. Then there is a unique formal power series $x=x(y)$ satisfying $x=y \phi(x)$. Further, if this series $x(y)$ is substituted into another formal power series $H$, then the resulting series satisfies

$$
\left[y^{n}\right] H(x(y))=\frac{1}{n}\left[x^{n-1}\right]\left\{H^{\prime}(x) \phi(x)^{n}\right\} .
$$

When $\phi(x)=\frac{1}{(1-x)^{r}}$ then the series $x(y)$ is the series $X(y)$ above. In this case,

$$
H(x(y))=\frac{1}{(1-X(y))^{s+1}}-1
$$

is the function on the right-hand side of (13). Lagrange inversion with $n+1$ in place of $n$ gives $H^{\prime}(x)=(s+1) /(1-x)^{s+2}$, whence

$$
\begin{aligned}
f(n) & =\left[y^{n+1}\right]\left(\frac{1}{(1-X(y))^{s+1}}-1\right) \\
& =\frac{1}{n+1}\left[x^{n}\right]\left\{\frac{s+1}{(1-x)^{s+2+(n+1) r}}\right\} \\
& =\frac{s+1}{n+1}\left(\begin{array}{c}
s+(n+1)(r+1) \\
n
\end{array}\right),
\end{aligned}
$$

proving (5).

\section{Probabilistic proofs}

Let $L$ be the set of points in $\mathbb{Z}^{2}$ defined by $L:=\left\{(i-1, j): 0 \leq i, 0 \leq j \leq b_{i}\right\}$. Fix $0<p<1$ and let $\Omega$ be the space of infinite sequences of 0 's and 1's, equipped with the 
product measure $\mathbb{P}$ making each coordinate 0 with probability $p$ and 1 with probability $q:=1-p$. With each $\omega \in \Omega$ we associate a lattice path beginning at the location $(-1,0)$, moving upward on step $k$ when $\omega(k)=1$, and moving right on step $k$ when $\omega(k)=0$, for each $k=0,1,2, \ldots$ If we let $S(k):=S(k, \omega)=\sum_{j=0}^{k} \omega(j)$, then a formal definition of the induced path is the sequence $\{(X(k), S(k)): k \geq 0\}$ of random vectors where $X(k):=k-S(k)$. If $\omega$ begins with a block of more than $b_{0} 1$ 's then the walk will be outside of $L$ when it takes its first step to the right, so to such an $\omega$ we associate the empty path.

Let $\tau(\omega)$ be the stopping time defined by $\tau:=\inf \left\{k \geq 0: S(k)>b_{1+X(k)}\right\}$. In other words, it is the first time $k$ that $X(k) \notin L$ (the barrier is exceeded). For each path, we now pass to the subsequence corresponding to the locations after moves to the right. Formally, define the random variable $M$ by

$$
M:=\sup \{X(k): k \leq \tau\}
$$

to be the farthest right extent of the path before exiting the barrier and define a random sequence $\mathbf{A}$ of length $M+1$ by

$$
A_{i}:=\min \{j: X(k)=(i, j) \text { for some } k \leq \tau\} .
$$

It is possible that $M$ is infinite (the barrier is never reached). It is also possible that $M=-1$, if initially there are $b_{0}+1$ upward moves, in which case, as remarked earlier, we set $\mathbf{A}:=\emptyset$, the empty path.

Proof of Theorem 3: To prove the inequality (6), it suffices to observe that for every sequence a of length $m+1$, the probability that $M=m$ and $\mathbf{A}=\mathbf{a}$ is equal to $p^{m+1} q^{b_{m+1}+1}$. Indeed, the event $\{\mathbf{A}=\mathbf{a}\}$ requires a specific sequence of values of $\omega(k)$ for $1 \leq k \leq m+b_{m}+2$, namely, upward moves to height $a_{0}$, then a move to the right, then upward moves to height $a_{1}$, then moves to the right, etc., ending with a move to the right that ends at $\left(m, a_{m}\right)$, followed by upward moves to height $b_{m+1}+1$; the total number of rightward moves is $m+1$ (remember, we started at $(-1,0)$ ) and the total number of upward moves is $b_{m+1}+1$, verifying the formula

$$
\mathbb{P}(\mathbf{A}=\mathbf{a})=p^{m+1} q^{b_{m+1}+1} .
$$

The event that $\mathbf{A}=\emptyset$ has probability $q^{b_{0}+1}$. Conditioning on this not occurring, we have

$$
1 \geq \mathbb{P}(M<\infty \mid M>-1)=\frac{\sum_{\mathbf{a}} p^{\ell(a)} q^{b_{\ell(a)}+1}}{1-q^{b_{0}+1}}=\sum_{\mathbf{a}} w(\mathbf{a})
$$

proving (6).

Evidently, equality holds if and only if $\mathbb{P}(M=\infty)=0$. This is the well known problem of whether a random walk can remain forever on one side of a barrier. An exact summability criterion is known for this under some regularity assumptions on the barrier. For example, in [Erd42, (0.13) and Theorems 1 and 3], Erdős proves a summability criterion in the case where $p=1 / 2$ and $n^{-1 / 2}\left(b_{n}-n / 2\right)$ is nondecreasing. The earliest version of such a test in the continuous time case is due to Petrowsky [Pet35]. 
Our theorem does not require results as sharp as these. It suffices to observe that if $\left(Y_{n}, Z_{n}\right)$ are the coordinates of $X_{n}$ then the strong law of large numbers implies that $Z_{n} / Y_{n} \rightarrow q / p$ almost surely. This implies that $\mathbb{P}\left(Z_{n} \geq b_{Y_{n}}\right.$ infinitely often $)=0$ when $\theta>q / p$, which implies that $\mathbb{P}\left(Z_{n} \geq b_{Y_{n}}\right.$ for some $\left.n\right)<1$, which implies $\mathbb{P}(M=\infty)>$ 0 . Conversely, if $\theta<q / p$, then the strong law of large numbers implies $\mathbb{P}\left(Z_{n}>(\theta+\right.$ e) $Y_{n}$ for sufficiently large $\left.n\right)=1$, which implies $\mathbb{P}(M=\infty)=0$. Finally, if $\theta=q / p$, the law of the iterated logarithm [Dur04, Theorem (9.7)] gives $Z_{n} \geq \theta Y_{n}+C \sqrt{Y_{n}}$ infinitely often almost surely for any $C$, which implies $\mathbb{P}(M=\infty)=0$ under the assumption $\left|b_{n}-\theta n\right| \leq C n^{1 / 2}$. This completes the proof of Theorem 3 .

\section{A question, and some acknowledgments}

We have not been able to generalize this combinatorial/probabilistic method to the situation where there is a lower, as well as an upper, barrier. Nonetheless the result of Kreweras cited above suggests that this may be possible.

Our thanks go to Mireille Bousquet-Mélou and Richard Stanley for citations to earlier work on this problem, and to Davar Khoshnevisan for citations to summability criteria for random walks.

\section{References}

[BMP00] M. Bousquet-Mélou and M. Petkovšek. Linear recurrences with constant coefficients: the multivariate case. Discrete Math., 225:51-75, 2000.

[Dur04] R. Durrett. Probability: Theory and Examples. Duxbury Press, Belmont, CA, third edition, 2004.

[Erd42] P. Erdős. On the law of the iterated logarithm. Ann. of Math., 43(3):419-436, 1942.

[FS08] Philippe Flajolet and Robert Sedgewick. Analytic Combinatorics. Cambridge University Press, 2008.

[Kre65] G. Kreweras. Sur une classe de problèmes de dénombrement liés au treillis des partitions des entiers. Cahiers du B.U.R.O., 6, 1965.

[Pet35] I. Petrowsky. Zur ersten Randwertaufgabe der Wärmeleitungsgleichung. Compositio. Math., 1:383-419, 1935.

[Pro88] R. Proctor. Odd symplectic groups. Invent. Math., 92:307-332, 1988.

[Sta99] Richard P. Stanley. Enumerative Combinatorics. Vol. 2. Cambridge University Press, Cambridge, 1999.

[Wil94] Herbert S. Wilf. generatingfunctionology. Academic Press, Boston, second edition, 1994. 